\title{
In Building Employee Tracking, Real Time Irregularity Detection and Warning (ETAW) System
}

\author{
Salman Afghani, Ayesha Sadique and Muhammad Ishfaq \\ $R \& D$ Department of Engineering and Sciences, \\ Army Public College of Management and Sciences (APCOMS), \\ Rawalpindi, Pakistan \\ dr.salman.afghani@gmail.com,ash_siddique@live.com,ishfaqjaved@gmail.com
}

\begin{abstract}
Since the very beginning of the industrial revolution the work-force (employees) is forced to perform repeated laborious tasks round the clock. To make things worse, most of the tasks are non-intellectual. With the passage of time while the employee is gaining experience and becoming more productive, he is losing human characteristics. And gradually transforming into a circus animal. Being inherently irregular and unpredictable employee (humans) require a system that can not only curb their irregularities but also, reminds them of their expected location via vocal announcements in real time. To meet these requirements, we present an efficient system that tracks the where about of employees in indoor environment. And keeps a compact tempo-spatial track records of each employee. It helps them to avoid irregularities in real time which otherwise may lead to accumulation of a considerable debt at the end of the month. The proposed system is both employee and organization friendly. The employee benefits because he/she is not deprived of human comfort level. And the organization benefits because the efficiency of employee is increase in real time.
\end{abstract}

Keywords: We would like to encourage you to list your keywords in this section

\section{Introduction}

In order to make the organization progressive, there must be a system that keeps a check over the employee's location and timings. In this paper, a system is presented that tracks employee's presence inside a building by recording his timings and locations, reminds him his expected location, in case of being late, automatically and warns him for irregularity, in real time.

Tracking and unpunctual behavior detection is the most recurring problem. While much research has focused on developing service architectures for location-aware systems, less attention has been paid to the fundamental and challenging problem of tracking mobile users, especially in in-building environments. The few efforts, which addressed this problem, have typically done so in the context of infrared (IR) wireless networks. The limited range of an IR network is a handicap in providing ubiquitous coverage. Moreover, GPS does not work well indoors, in cluttered urban environments or under dense foliage. To avoid these limitations, we focus on RF wireless networks in our research. Our goal is to implement a data networking RF system with accurate employee tracking capability and cost effectiveness. 


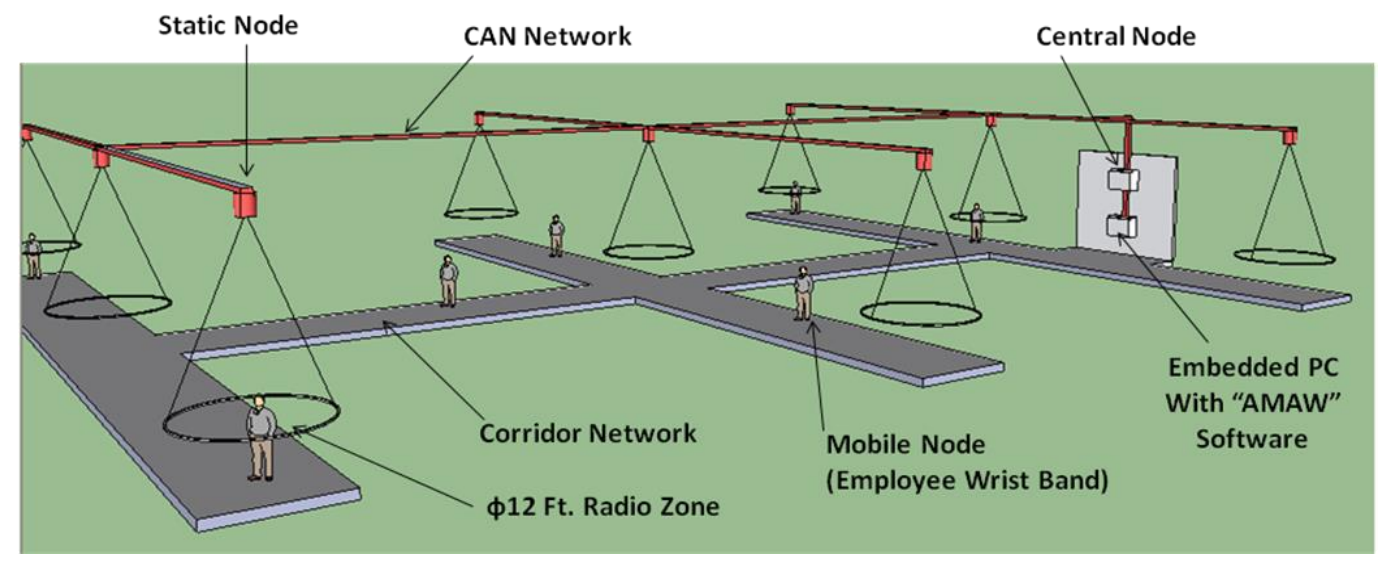

Figure 1. Physical Layout of ETAW System in structured environment

\section{Description of "ETAW" System}

The in building employee tracking and real time irregularity warning system "ETAW" is constituent of the following Functional components:

- $\quad$ Short Range RFID (Employee to CAN network Radio communication)

- CAN network (RS-485 based Twisted Pair)

- $\quad$ Embedded PC based Attendance Marking and Absence Warning (AMAW) software and Employee Attendance Database

The functional description of the ETAW system is as follows:

1. Every employee of the ETAW enabled organization carries a miniature duplex RFID unit around his/her wrist. And every RFID unit has its own unique ID as-signed to the name of the employee wearing it.

2. The RFID unit stays dormant (does not transmit) until the employee enters the radio zone (12ft radius) of any node in the CAN network deployed over the building of "ETAW" embedded organization.

3. The moment the RFID unit (mobile mode) of the employee receives a radio syncbroadcast signal from a static node of the CAN Network, it communicates its ID to static node.

4. The received ID is 'Location tagged' by static node by complementing the employee ID, with its own ID.

5. This info. Carrying 'Personal ID' along with the 'Location ID' is communicated by the node through CAN network to the Central node.

6. The central node further adds a "Time tag" to the original data and stores it into a $2 \mathrm{D}$ array called 'Time Frame'. At this point the employee is represented by 3 byte.

- Individual ID

- Location tag

- Time tag 
Hence the central node receives info. About the where about of employee from 4 corners of the 'ETAW' enabled building through 16 static nodes evenly places across the corridors of the building.

7. Until now we were discussing about the Data Acquisition part of the "ETAW" system. The analysis and synthesis of the Acquired Information takes place in a embedded 'PC-onboard' device, which is interfaced to the central node via USB.

8. It is to be noted that uptill now Data Moved up from the Mobile Node (Employee) to CAN network and from there on to the embedded PC. The embedded PC runs a software called "AMAW" (Attendance Marking and Absence Warning). As shown in Figure 7 (Data structure and Data Flow for Warning Generation).

This software analyzes the current Time Frame by comparing it with the corresponding Time Table in the daily scheduler of the organization. This is how the attendance of each employee is checked.

9. The monthly data base of the organization is updated regularly by saving called attendance of the employee in a 2D array called "Attendance Frame". This "Attendance Frame" is stored into the "Employee attendance Data Base" for record keeping. From this point Data moves down from PC towards Mobile Nodes. As shown in Figure 6 (Data Structure and Data Flow for Employee Tracking)

10. A Synthesis of the Acquired Information is produced by the "AMAW" Software by entering the ID's of absent Employee's in a 2D array called "Warning announcement Frame".

11. This "Warning Announcement Frame" is communicated back to the Centered Node via USB.

12. The central node further transfers the "Absence Warning Announcement" Frame down to the 16 Static Nodes of the CAN network.

13. A Voice Synthesizer along with a Audio system inte-grated into each Static Node then announces the Names of the Irregular Employees repeatedly (3 times) at locations where they were Last Located. As shown in figure Figure 6 (Data Structure and Data Flow for Employee Tracking)

14. The whole Procedure of tracking attendance marking and absence warning is repeated continuously at the rate of 1 Attendance Frame every 10 seconds.

\section{Subsystems of ETAW and Their Features}

\subsection{Central Node:}

The Central Node consists of two functional blocks:

a. Centre to PC com. Section (CUSB): Responsible for interchanging information about the employees with the computer. In other words CUSB acts as bridge between the Hard and Soft sections of our system (ETAW).

b. Center to Static Node com. Section (CNET): Responsible for interchanging information about employees with the CAN Network of static nodes. In other words CNET is the master of the CAN Network. 


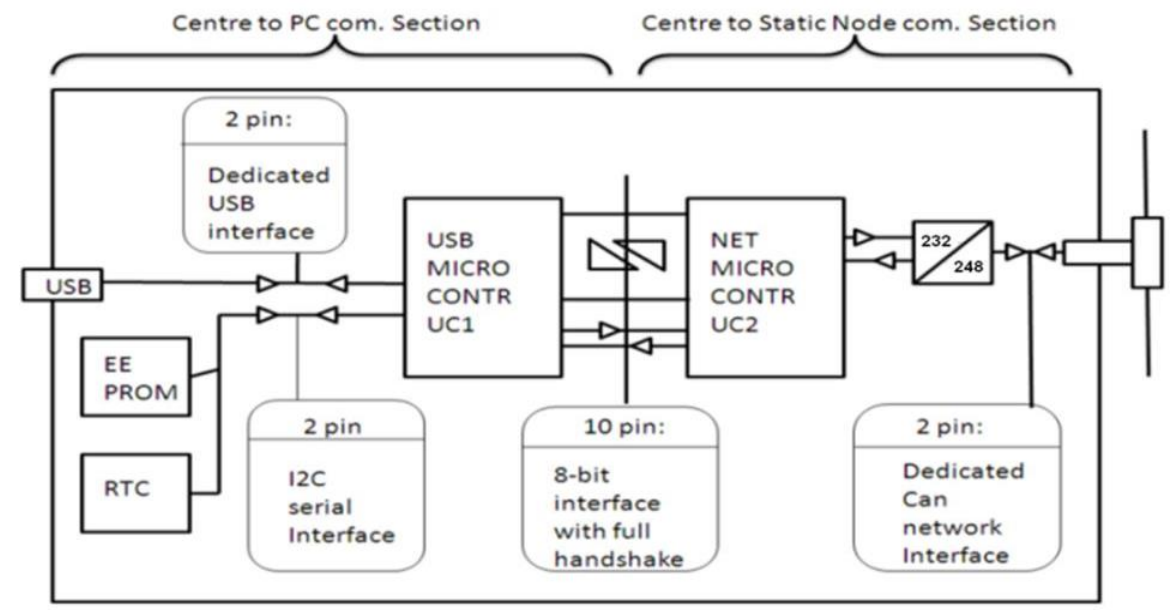

Figure 2. Central Node

\subsection{Static Node:}

Situated at strategic locations of the ETAW enabled building, the static node is responsible for:

- $\quad$ Radio com. with the mobile nodes worn by the Employees as RFID wrist band.

- $\quad$ CAN network based com. With the central node

- Audio Announcements via speech synthesizer.

The static node is functionally bifurcation into two sections:

a. Static Radio Section

b. Static CAN+Audio Section

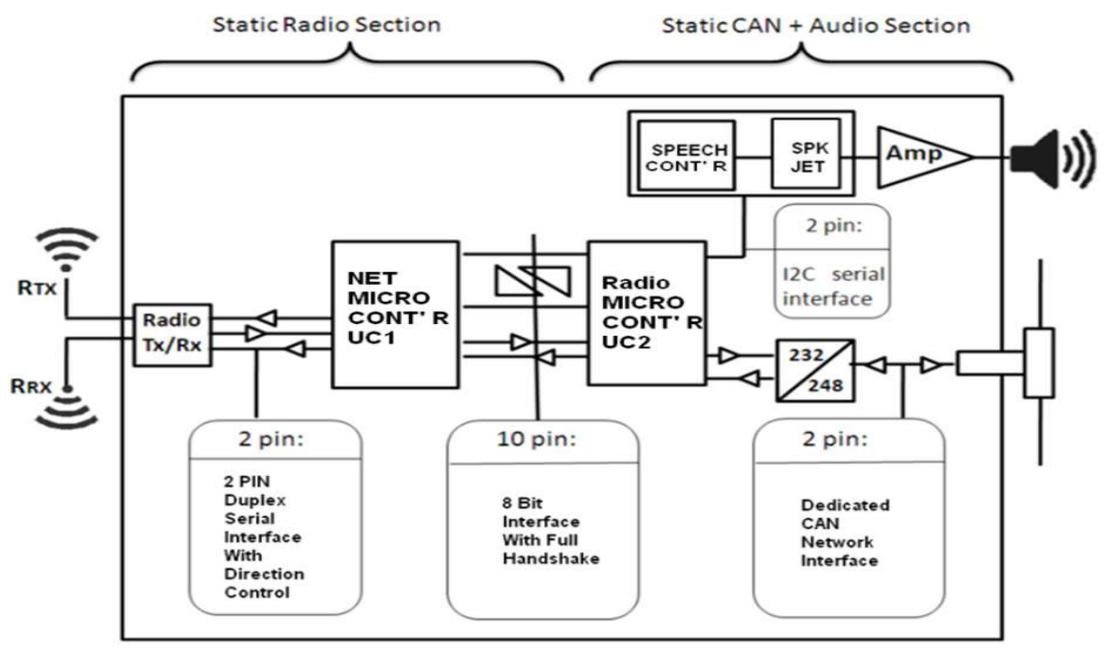

Figure 3. Static Node 


\subsection{Mobile Node}

Worn as a wrist band RFID based device the mobile node consists of three functional blocks:

- Digital Duplex Radio (Tx/Rx) Module

- $\quad$ Radio com. protocol running microcontroller with employee ID

- $\quad$ Random number generator for facilitating Probabilistic Queuing Technique (PQT) for radio phase alignment between static node and the mobile node itself.

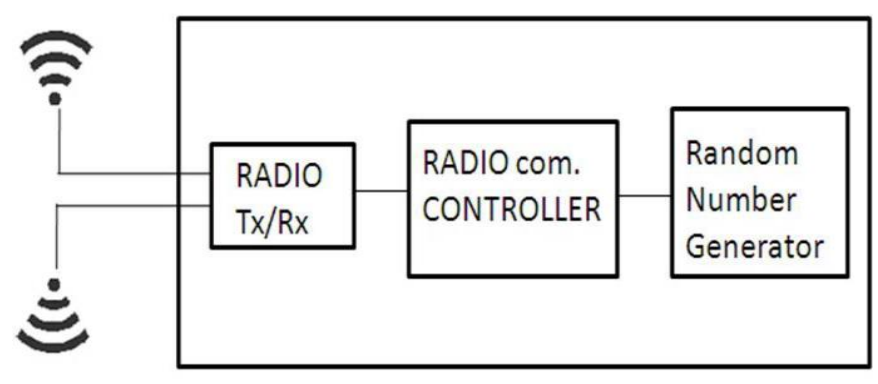

Figure 4. Mobile Node

\subsection{Embedded PC based Attendance Marking and Absence Warning (AMAW) software and Employee Attendance Database:}

The analysis and synthesis of acquired information about the ware about of the employees takes place in an embedded PC. The embedded PC runs a software called "AMAW". This software marks the attendance of the employees and generates warning announcements for irregular employees in real time. In addition the embedded PC maintains.

\section{Working of the System}

\subsection{Internal Data Exchange}

A Voice Synthesizer along with a Audio system integrated into each Static Node the announces the Name of the Irregular Employee at location where they were Last Located.

Central Node: The 2 halves (CNET and CUSB) and of cen-tral node exchange data among them salves with in the central node itself, such that.

i) A Frame of 96 bytes containing the ID's Register in 16 radio zones transferred from CNET to CUSB.

ii) CUSB Time Tagged the frame (96Bytes) using the onboard RTC.

iii) A string of 16 bytes same containing space tagged ID's is of irregular employees (the rest empty) is transferred by CUSB to CNET.

Static Node: The 2 halves (SNET and SRAD) of static node exchange data among themselves, within the static node itself (all 16 static nodes), such that

i) A string of 6 bytes; same as the ID's of mobile nodes currently detected in the radio zone of a static node (SRAD). Is transferred to the Net Section (SNET) of the static node. 
ii) After Discarding the ID's with space tags not be-longing to that of the static node itself.

iii) The net section of static node transfers the ID's with space tags belonging to itself to the radio section (SRAD) for Audio Announcement.

\subsection{At This Point the ETAW Splits into Two Independent, Concurrently Functional Modules}

$>$ SOFT ETAW

$>$ HARD ETAW

4.2.1. SOFT ETAW: This module comprises of the USB sec-tion of the central node and the master PC resident "AMAW" software. The soft ETAW functions in the following manner:

A 3D array of $850 \mathrm{~Kb}$ carrying IDs of mobile nodes detected in 16 radio zones is transferred from CUSB to PC via USB where it is stored in a database. And a PC resident software scans the most recently captured time frame of mobile IDs. And compares the temporo - spacial info. of each ID with that in the corresponding daily employee scheduler of the organization. If the employee is present in the right place and at the right time. Or was absent for an acceptable period of time. Then the software ignores the ID and moves on to the next ID. And the same procedure is followed for the rest of the 95 IDs. But if the temporospecail info. does not tally with the scheduler, then the employee is declare irregular and a warning flag is set against his ID.

Eventually a 2D array of 96 bytes ( 1 time frame) containing latest location info. of irregular IDs. Is communicated back to that particular static node where the ID was last detected.

4.2.2 HARD ETAW: The hard ETAW comprises of the net section of the central node, the static nodes (SNET) and the mobile nodes.

The Hard ETAW performing the following sequence of operations:

\subsubsection{Net Talk:}

Central Node: The central node addresses one by one each of the 16 static nodes via I2C serial interface and exchanges data with them. Data about the timely where about of the mobile nodes is collected by the central node. Whereas irregularity warning data generated by the PC resident software "IEDAWS" is relayed down to the static nodes for audio announcement.

Note: IEDAWS stands for "Irregular Employee Detection and Real Time Warning Software"

Static Node: Each of the 16 static nodes waits for a Net-Talk session with the central node. When addressed via I2C serial interface by the central node, each static node transfers the ID of mobile node it captured within its radio zone to the central node. 
And reads in irregularity warning data from the central node. Which is later converted into allophonic speech by the speech processor, and announced via audio.

\subsubsection{INIT_RPT:}

Due to the fact that probabilistic queuing technique is being used, the queue turn number( NQT ) of a couple or more mobile nodes might coincide and as a result corrupt each others data in one hexagonal radio com. session. Therefore full radio com. session is repeated thrice by maintaining a radio repetition counter (CRR).

Static Node: Initialize radio repetition counter $(C R R=00)$

Mobile Node: Initialize radio repetition counter $(C R R=00)$

\subsubsection{Radio Sync broadcast:}

Static Node: Each slave emits a synchronization signal in its radio zones to initiates a radio-com. Session with mobile nodes present inside its radio zone.

Mobile Node: Each Mobile node only listens, waiting for phase synchronization. Radio signal from any of the static nodes. And the moment phase synchronization radio signal is received, the mobile nodes phase shift itself to get aligned with the static node.

\subsubsection{Radio Sync Signal Authentication:}

Mobile Node: If received radio data is not corrupt and its system ID is verified; and if the received data carries the ID of a static node. Only then the mobile node engages itself into a radio-com session.

\subsubsection{Random Number Generator:}

Mobile Node: if the radio synchronization signal is authentic, the mobile node is ready for a radio com session. But there could be more than one mobile node in one radio zone. Therefore a asynchronous random queuing technique is incorporated into the system. According to which the mobile node reads a 3 bit ( 1 of 6) random No generator and assigns the random No to a token in [NQT].

\subsubsection{Hexagonal Radio-com Sequencer:}

Mobile Node: Each mobile node initializes a six step radio com turn counter [CRET $=00$ ]

\section{COMPARE:}

Run radio com. timer $[\mathrm{TRC}=\mathrm{RUN}]$ and compare radio Tran. Rec. Segment Counter with queue turn number (Is CTRS $=$ NQT ?) .

EQUAL: If this is the turn to Talk (If CRCT $=$ NQT,) then mobile node transmits its ID to static nodes. And waits for conformation massage from static node, while the radio com. session timer is still running. When radio com. session time is over, it is checked if mobile node is in a new radio zone ? (IF old $=0$ ?) 


\section{OLD:}

$(\mathrm{F}$ old $=1)$ If mobile is still in same radio zone, then just wait for the radio com. Segment timer to be over. And go to INC_CTRS.

\section{NEW:}

$(\mathrm{F}$ old $=0)$ If mobile node has just entered a new radio zone, then mobile node transmit its ID to host static node.

And wait for conformation message from static node, while the radio com. Segment timer is still running (TRS not $=00$ ).

CONFIRMATION: If confirmation message is received, then the mobile node declared the present radio zone as OLD and sets a flag ' $\mathrm{F}$ old $=1$ '. And goes to INC_CRCT .

NO CONFIRMATION: If confirmation is not received, then the mobile node takes no action. Just wait for radio com. Segment timer to be over (TRCS $=00$ ). And goes to INC_CRCT .

NOT EQUAL: If CRCT not $=$ NQT, then this is not the turn to Talk (this turn is skipped). The mobile node waits for the radio com. Segment timer to be over (TRS = 00). And goes to INC_CRCT .

\section{INC_CTRS:}

Increment Radio Tran. Rec. Segment counter is incremented (CTRS+1). And Check if this was the last segment of the hexagonal radio com. Sequencer ? (CTRS=6 ?)

YES: A full radio com. Session with mobile nodes in 16 radio zone is completed. But some of the mobile nodes could have over lapped with their randomly acquired queue turn numbers. Therefore full radio com. Session will be repeated. Go to "REPEATER".

NO: The radio trans-Rec segment (CTRS) is not equal to Queue Turn Number (NQT). Goto "COMPARE"

Graphical representation of Hexagonal Radio-com Sequencer is shown in Figure 5. 


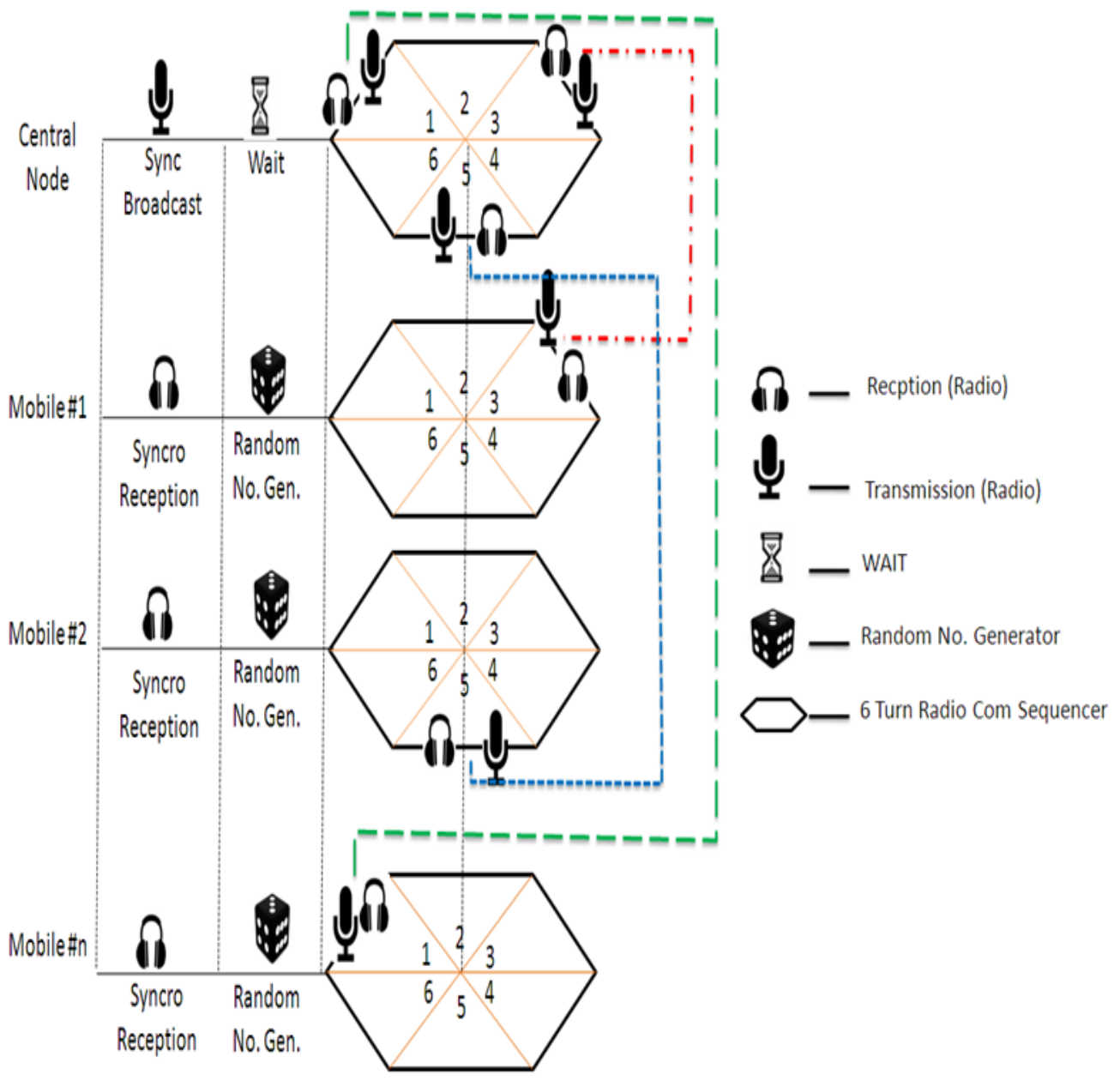

Figure 5. Hexagonal Radio-com Sequencer

\subsubsection{Repeater:}

Increment radio repeat counter $[\mathrm{CRR}+1]$

If CRR not $=3$, then full radio com. Session needs to be re-peated because some of the mobile node could have overlapped with their randomly acquired queue turn number. Go to step 'Radio Sync Broadcast'.

If $\mathrm{CRR}=3$, then 3 repeated full radio com. Session have been conducted for all mobile nodes in all 16 radio zones. And one time frame of 2D array for 96 IDs is ready. 


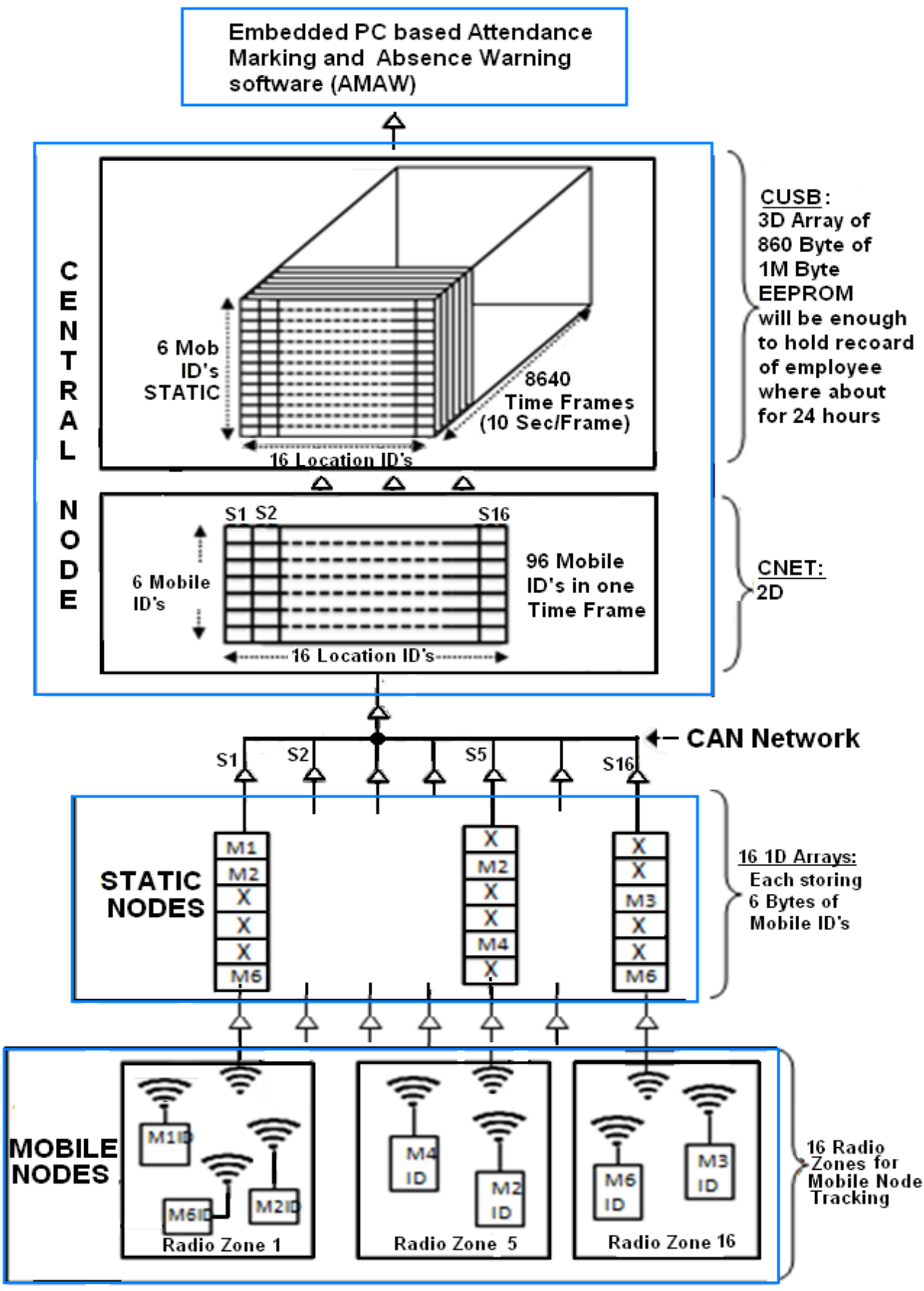

Figure 6. Data Structure and Data Flow for Employee Tracking 


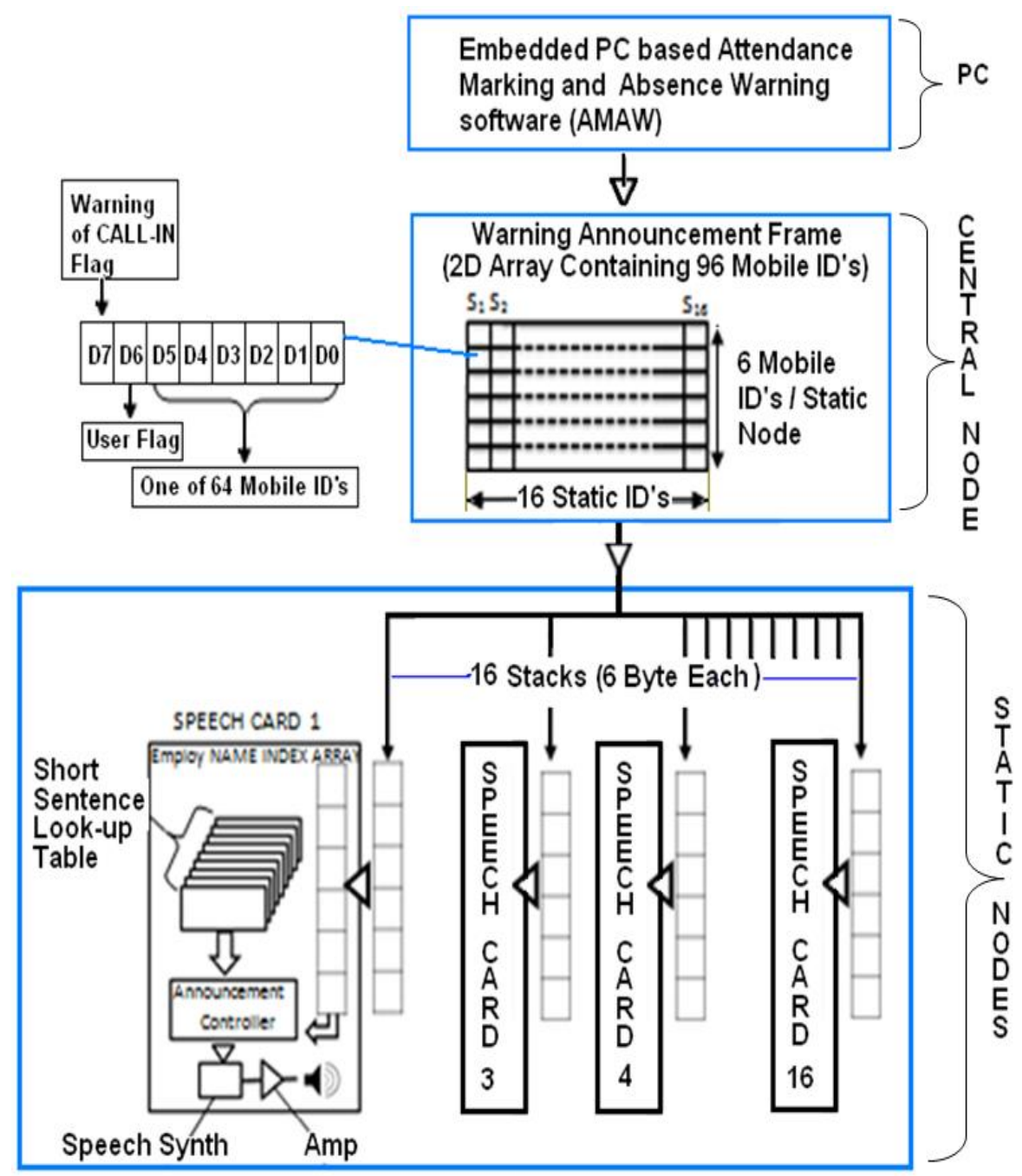

Figure 7. Data structure and Data Flow for Warning Generation

\section{Conclusions}

The ETAW system prototype was developed by using readily available low cost off the shelf components and devices. The system was deployed on one floor of the engineering block of our institution using 16 static nodes. The system performed reliably with the acception of few glitches and bugs in the AMAW software.

ETAW is easily scalable for a building with many floors with large spread and span of corridors. In developing countries where the concentration of HR is high but due to financial constraints advanced high end, costly automation in not in applicable. This is where an efficient, low cost system like ETAW can improve the performance of an institution, organization or industry keeping in mind the comfort level of the employee as human being and the limited budget as well. Airports, Hospitals, Educational Institutions, Warehouses, Banks and other large commercial setups can greatly benefit from ETAW system. 


\section{References}

[1] Harter and A. Hopper, "A Distributed Location System for the Active Office", IEEE Network, (1994) January.

[2] P. Bahl and V. N. Padmanabhan, "RADAR: An in-building RF-based User location and tracking system", Proceedings of IEEE Infocom 2000, (2000), pp. 775-784.

[3] Fritsche and A. Klein, "On the Performance of Hybrid GPS/GSM Mobile Terminal Tracking", IEEE ICC Workshops, (2009) June, pp. 1- 5.

[4] Zhang, J. Ma, Q. Chen and L. M. Ni, "An RF-based system for tracking transceiver-free objects", in IEEE PerCom'07, (2007), pp. 135-144.

[5] Galileo European Satellite Navigation System, http://ec.europa.eu/dgs/energy_transport/galileo/ index_en.html.

[6] K. Lorincz and M. Welsh, "MoteTrack: A Robust, Decentralized Approach to RF-Based Location Tracking", In Proceedings of the International Workshop on Location and Context-Awareness, (2005).

[7] R. Azuma, "Tracking Requirements for Augmented Reality", Communications of the ACM, vol. 36, no. 7, (1993) July, pp. 50- 51.

[8] N. B. Priyantha, "The cricket indoor location system", Ph.D. Thesis, Massachusetts Institute of Technology, (2005) June.

[9] T. W. Christ and P. A. Godwin, "A Prison Guard Duress Alarm Location System", Proc. IEEE International Carnahan Conference on Security Technology, (1993) October.

[10] L. Reggiani, M. Rydstr"om, G. Tiberi, E. G. Str"om and A. Monorchio, "Ultra- wide band sensor networks for tracking point scatterers or relays", in Sixth International Symposium on Wireless Communication Systems2009 (ISWCS-09), (2009) September.

[11] M. Azizyan, I. Constandache and R. R. Choudhury, "Surround Sense: mobile phone localization via ambiance fingerprinting", ACM MobiCom, (2009) June, pp. 261-272.

[12] M. A. Kanso and M. G. Rabbat, "Efficient detection and localization of assets in emergency situations," 3rd Intl. Symposium on Medical Information\& Communication Technology (ISMICT), (Montr'eal, Qu'ebec), (2009) February.

[13] The Active Badge system, http://www.cl.cam.ac.uk/research/dtg/attarchive/ab.html.

[14] The Bat ultrasonic location system, http://www.cl.cam.ac.uk/research/dtg/attarchive/bat.html.

[15] X. Tian, Y. Miao, T. Hu, B. Fan, J. Pan and W. Xu, "Maximum Likelihood Estimation Based on Time Synchronization Algorithm for Wireless Sensor Networks", ISECS International Colloquium on Computing, Communication, Control, and Management (CCCM), (2009) August, pp. 416-420.

[16] Y. Gu, A. Lo and I. Niemegeers, "A Survey of Indoor Positioning Systems for Wireless Personal Networks", IEEE Communications Surveys \& Tutorials, vol. 11, no. 1, (2009), pp. 13-32.

\section{Authors}

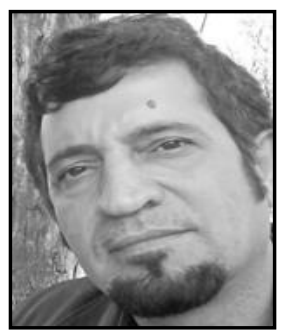

Salman Afghani was born in 07/20/1958 in Pakistan and he is Professor, $\mathrm{PhD}$, Advancedman machine systems, MPhil, Indus-trial automation, MS Mech Engg. He is working as HOD of R\&D Department of Engineering Sciences at Army Public College of Management and Sciences (APCOMS), Rawalpindi Pakistan. 


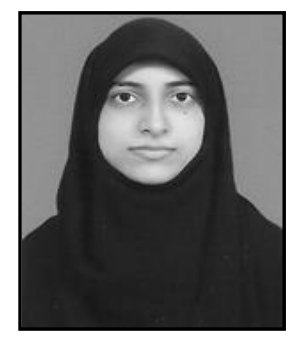

Ayesha Sadique is BSc. Electrical (Telecom) Engineer. She did her graduation from Army Public College of Management and Sciences. Her interests are control automation, digital image processing and control system.

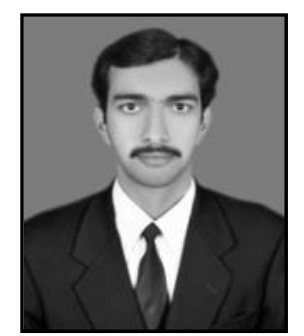

Muhammad Ishfaq Javed is Electrical (Electronics) engineer from Army Public College of Management and Sciences (AP-COMS), Rawalpindi, Pakistan. His research interests are control and automation, man machine interface, Robotics and Microcontroller Programming. 
International Journal of Advanced Science and Technology Vol.66 (2014) 